\title{
TEMPORAL RELATIONSHIP BETWEEN SNIFFING AND THE LIMBIC $\theta$ RHYTHM DURING ODOR DISCRIMINATION REVERSAL LEARNING ${ }^{1}$
}

\author{
FOTEOS MACRIDES, ${ }^{2}$ HOWARD B. EICHENBAUM, ${ }^{*}$ aND WILLIAM B. FORBES \\ Worcester Foundation for Experimental Biology, Shrewsbury, Massachusetts 01545 and ${ }^{*}$ Department of Biology, Wellesley \\ College, Wellesley, Massachusetts 02181
}

Received May 24, 1982; Revised July 26, 1982; Accepted August 24, 1982

\begin{abstract}
The temporal relationship between sniffing and the limbic $\theta$ rhythm was studied in rats during odor discrimination reversal learning. The $\theta$ rhythm was monitored as rhythmic slow wave activity (RSA) in the dorsal hippocampal formation, and cyclic nasal airflow (sniffing) was monitored with a thermocouple in the nasal cavity. The training procedures required animals to perform a sequence of whole body locomotion toward one wall of an arena, followed by investigatory sniffing of stimuli through a port while otherwise standing still. Hippocampal RSA was present reliably during the periods of investigatory sniffing. Analyses based on the fast Fourier transform (FFT) demonstrated that this RSA tended to be lower in frequency and amplitude than RSA which occurred during locomotory approach. Other analyses based on the FFT were developed to characterize the nature and parameters of the temporal relationship between rhythmic sniffing and hippocampal RSA as a function of the dominant sniffing frequency during the periods of stimulus sampling. The phase difference between sniffing and RSA tended to vary linearly with frequency so as to maintain a preferred latency relationship between the onset of each sniff cycle and a particular phase of the hippocampal RSA. The phase of RSA to which sniffing was related differed across animals and was correlated with electrode position relative to the phase reversal layers within the hippocampal formation. These results therefore are consistent with the interpretation that, during the periods of stimulus sampling, the sniffs were being timed to maintain a preferred latency relationship with the pacemaker activity which drives the $\theta$ rhythm and the recorded RSA.

The consistency with which the animals exhibited the preferred latency relationship varied during the course of training. Across animals, this correlation between sniffing and $\theta$ activity was consistently high during the trials which immediately preceded the achievement of criterion level performance, and the correlation was reduced during the criterion run and/or subsequent trials of overtraining. Thus, the tendency of the animals to exhibit this relationship was not associated specifically with correct performance. Rather, the correlation tended to be highest, when the animals were most likely to be evaluating the behavioral relevance of stimuli and were in the process of modifying their responses to those stimuli. The timing of investigatory sniffs as a function of $\theta$ cycle phase may be important for the neural processing of sensory and/or motor information of relevance for response modification.
\end{abstract}

The mammalian limbic system exhibits a pronounced 4- to $12-\mathrm{Hz}$ electrographic rhythm during a variety of behavioral and drug states (cf., Vanderwolf, 1975; Komisaruk, 1977). This neural rhythm commonly is referred

\footnotetext{
'This work was supported by National Institutes of Health Research Grants NS12344 and AG00779, National Science Foundation Research Grants BNS77-24405 and BNS78-06248, and National Science Foundation Equipment Grants PRM-COP81-6784 and BNS78-18113. We thank Karen Shedlack and Paul Murphy for technical assistance.

${ }^{2}$ To whom reprint requests should be sent at the Worcester Foundation for Experimental Biology, 222 Maple Avenue, Shrewsbury, MA 01545 .
}

to as $\theta$ activity, although its upper frequency range extends into the $\alpha$ band. $\theta$ activity has been recorded as rhythmic single unit or slow wave activity in numerous limbic and related structures of the forebrain and is prominent in waking animals during active exploration of the environment (cf., Komisaruk, 1977). The limbic $\theta$ rhythm can be monitored readily as rhythmic slow wave activity (RSA) in the hippocampal formation (cf., Winson, 1974). Its pacemaker activity is thought to originate in neurons of the medial septum-diagonal band complex (cf., Petsche et al., 1962, 1965), and its occurrence and frequency appear to be regulated through multisynaptic 
pathways originating in the pontine reticular formation (Vertes, 1981). The presence of hippocampal RSA in electroencephalographic records has been associated with voluntary movement (Vanderwolf, 1971), spatial guidance (O'Keefe and Nadel, 1978), attention (Bennett, 1975), and learning (Adey et al., 1960; Landfield et al., 1972; Berry and Thompson, 1978).

Many of the previous attempts to characterize the behavioral correlates of the limbic $\theta$ rhythm have examined the presence or absence of $\theta$ activity in electroencephalographic records but have not focused specifically on its rhythmic qualities. However, temporal correlations between individual cycles of the $\theta$ rhythm and relevant sensory or motor events have been reported. For examplc, during operant conditioning, rats are more likely to depress or release a bar at particular phases of the $\theta$ cycles (Semba and Komisaruk, 1978; Buño and Velluti, 1977). Komisaruk (1970) also reported that rats commonly exhibit entrainments between the limbic $\theta$ rhythm and the repetitive motor components of exploratory sniffing behavior (fixation of the head and neck, inhalation, and protraction of the mystacial vibrissae; cf. Wclker, 1964). Lesions of the medial septum-diagonal band complex which eliminate hippocampal RSA can affect the coordination of motor activity associated with investigatory sniffing but do not eliminate rhythmic sniffing behavior (Gray, 1971). 'I'he pacemaker activity which drives the $\theta$ rhythm thus may play a role in the temporal regulation of exploratory sniffing behavior but does not drive the sniffing rhythm directly (cf., Macrides, 1975). Komisaruk (1970) has pointed out that, during entrainments between the two rhythms, the sensory activity evoked through the exploratory motor acts in turn would be correlated with $\theta$-related unit activity in the forebrain, and he speculated that such correlations might provide a temporal basis for modulating the central processing of sensory evoked activity so as to elicit appropriate behavioral or hormonal responses (cf., Komisaruk and Beyer, 1972; Scott, 1977). Consistent with this speculation, Komisaruk (1977) subsequently observed that the ability of olfactory bulb shocks to elicit hypothalamic unit responses varies according to the phase of the $\theta$ cycle during which the stimulation is delivered. Hippocampal excitability in response to stimulation of the entorhinal cortex similarly has been shown to be phase locked to the $\theta$ rhythm (Rudell et al., 1980).

Komisaruk (1970) described the entrainments between sniffing bouts and the $\theta$ rhythm in rats as increases in the frequency of inhalation and vibrissal sweeping to that of the ongoing $\theta$ activity. He noted that the phase relationships between these exploratory movements and the $\theta$ rhythm could vary from one entrainment to another. We subsequently examined the relationships among inhalation, vibrissal movements, and hippocampal RSA in male hamsters during bouts of exploratory sniffing toward localized odor sources in an open field (Macrides, 1975; Youngs et al., 1978). Our analyses demonstrated that sniffing and vibrissal sweeping are repetitive at the same frequency as hippocampal RSA more often than would be expected by chance on the basis of their respective frequency distributions. In addition, our analyses indicated that, during such entrainments, these motor acts exhibit a preferred temporal relationship to cycles of the RSA. Specifically, the phase differences between the motor components of exploratory sniffing behavior and the hippocampal RSA varied with frequency in a manner suggestive of a preferred latency relationship between the timing of the exploratory movements and particular phases of the $\theta$ cycles. The incidence of entrainments also varied in a manner which suggested that they might play a role in the central regulation of behavioral or hormonal responses. Entrainments tended to occur during the early presentations of arbitrary pure chemical stimuli, when these stimuli were relatively novel, and occurred most reliably during presentations of biologically relevant stimuli such as the vaginal discharge of female hamsters. The latter stimulus has been shown to facilitate sexual arousal in male hamsters (e.g., it elicits copulatory behavior toward female surrogates; Murphy, 1973; Macrides et al., 1977) and, under appropriate circumstances, elicits a neuroendocrine response resulting in an elevation of circulating testosterone levels (Macrides et al., 1974).

The experiments in this report had several goals. We hoped to develop a behavioral paradigm which would enable us to examine the relationship between rhythmic sniffing and the limbic $\theta$ rhythm under conditions in which movements other than those associated with investigatory sniffing behavior were minimized. Our paradigm was designed to encourage a sequence of locomotory approach, followed by sniffing of stimuli through a port while otherwise standing still. We further wished to determine whether rats, like hamsters, exhibit a preferred temporal relationship between sniffing and the $\theta$ rhythm during entrainments between the two rhythms and to develop quantitative procedures for characterizing the parameters of this relationship. Finally, we sought to develop training procedures and statistical analyses which would enable us to study the possible importance of this relationship for the acquisition of learned responses.

\section{Materials and Methods}

Experiments were performed on 13 male Long-Evans rats (300 to $400 \mathrm{gm}$ ) obtained from Charles River Breeding Laboratories, Inc. (Wilmington, MA). The behavioral paradigm was adapted from a previous odor discrimination task (Eichenbaum et al., 1980). Seven of the animals were used to evaluate the suitability of the behavioral paradigm, and only qualitative assessments of the relationship between sniffing and hippocampal RSA were made using graphic display and averaging procedures adapted from our previous, open field study in hamsters (Macrides, 1975). This report concentrates on 6 rats (RM-8 to RM-13) whose data also were subjected to quantitative computer analyses employing the fast Fourier transform (FFT).

Recording procedures. Nasal airflow was monitored with a thermocouple (Control Products Co., Chicago, IL) embedded in the tip of a stainless steel sheath $(0.25 \mathrm{~mm}$ outer diameter) and lowered vertically into the nasal passage through a 27 gauge guide tube implanted in the nasofrontal suture. The thermocouple assembly was positioned immediately rostral to the olfactory turbinates. 
The output of the thermocouple was inverted so that inhalation (cooling of the probe) produced a positive voltage swing, and the depth of the probe was adjusted for maximum voltage swings during sniffing. The onsets of individual sniff cycles were defined arbitrarily as the positive zero crossings in the $\mathrm{AC}$-coupled signal from the thermocouple or as the zero phases of sine waves fitted to the raw data using the FFT (see below).

Slow potentials were recorded from the dorsal hippocampal formation with a bipolar, twisted pair electrode (125- $\mu \mathrm{m}$-diameter stainless steel wires, Teflon-coated, deep tip noninverting) implanted with the skull surface leveled. A stainless steel skull screw over the frontal pole served as an indifferent electrode. The vertical tip separation and stereotactic coordinates of the implanted electrode were varied across animals to investigate possible differences in the relationship between sniffing and hippocampal RSA as a function of recording location. Electrodes were not placed in the ventral hippocampal formation in order to avoid possible contamination of the RSA with volume-conducted slow waves from the piriform cortex. Small electrolytic lesions were made after completion of the behavioral testing to facilitate histological localization of the electrode tips. During testing, recordings were made with unity gain fluid effect transistor (FET) followers attached to the skull in order to eliminate possible cable artifacts. The cable leads were connected through a commutator/counterbalance assembly. The electrode and thermocouple signals were amplified (1.5- to $100-\mathrm{Hz}$ bandpass) and recorded on magnetic tape along with trial event markers.

Behavioral apparatus and training. The training chamber consisted of a $48 \times 72 \mathrm{~cm}$ metallic arena with a grid floor and slanted walls (Fig. 1). The chamber was floating electrically and was contained within a soundattenuating shielded box. The rat was viewed inside of the box with the use of a closed circuit video monitor. A 2.5 -cm-diameter port was located in the center of one wall of the training chamber and a water delivery cup was positioned on the floor below the port. Odor stimuli, phenethyl alcohol (Eastman) and geraniol (Givaudan), were diluted to $4 \%$ of saturation in a flow dilution glass and Teflon olfactometer and were presented through the inner barrel of a concentric two-barrel nozzle positioned outside of the chamber near the center of the port. Odors were removed actively through the outer barrel by aspiration at four times the $500 \mathrm{ml} / \mathrm{min}$ presentation rate. In addition, an overhead fan with a charcoal filter ventilated the shielded box continuously. The trial sequence and presentation of stimuli were controlled electronically.

After a 1-week postsurgical recovery period, each rat was deprived of water for $24 \mathrm{hr}$ and thereafter received water only during the daily training sessions and during a 15-min free drinking period following each session. The rat initially was shaped to insert its nose into the port and break a photocell beam for at least $2 \mathrm{sec}$ in order to obtain a $0.05-\mathrm{ml}$ water reward. During an 8 -sec minimum intertrial interval, a guillotine door prevented access to the port. The rat subsequently was shaped to initiate trials by walking across the center of the arena, thereby breaking another photocell beam, and a 2-sec interval was imposed between the time of trial initiation and the raising of the guillotine door. Shaping was performed without the presentation of odor stimuli and was continued until the rat consistently and rapidly initiated trials, approached the port, and held its nose in the port for at
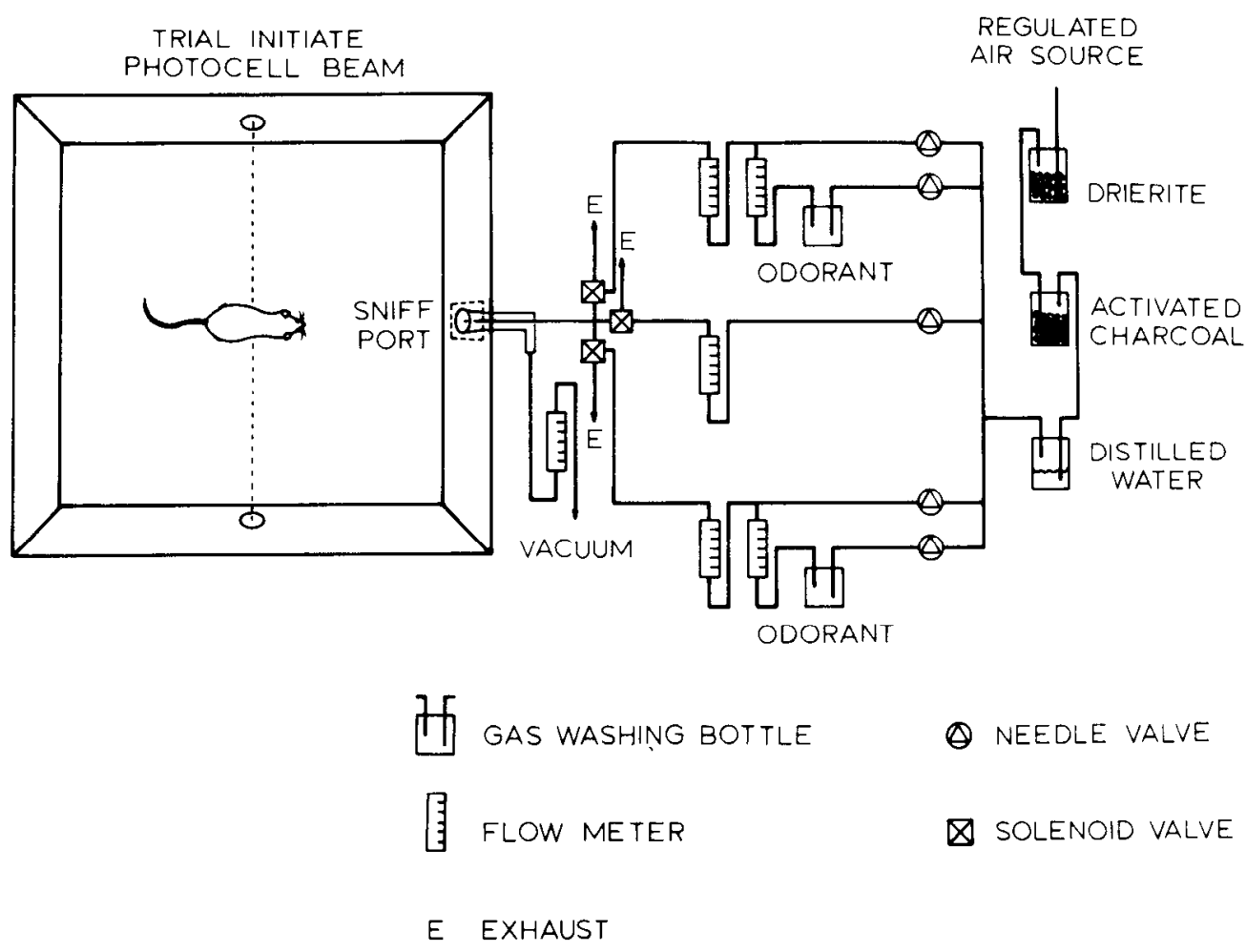

Figure 1. Schematic diagram of the training chamber and olfactometer. See the text for description. 
least $2 \sec (\mathrm{R}+)$. Then odor stimuli, arbitrarily designated as $\mathrm{S}+$ and $\mathrm{S}-$, were presented in random order on successive trials. Rewards were given only for the $R+$ response in the presence of $\mathrm{S}+$. Initially, nearly all responses were $\mathrm{R}+$, but eventually, each rat would learn to withdraw its nose in less than 2 sec $(\mathrm{R}-)$ in the presence of S-. When a performance criterion of $90 \%$ correct in 20 consecutive trials was reached, an additional 40 trials were allowed and then the valences of odor stimuli abruptly were reversed. Reversal training was continued until the $90 \%$ performance criterion again was achieved and at least 40 additional trials (overtraining) were completed. On each day, the rat was trained until it ceased initiating trials for $10 \mathrm{~min}$ or until it had completed 250 trials. Several of the animals were trained on additional reversals until they had acquired a reversal learning set.

In our initial experiments, the lowering of the guillotine door was triggered immediately after a rat removed its nose from the port. This procedure appeared to generate false $\mathrm{R}$ - responses in that the rats occasionally continued to sniff vigorously at the port and thereafter displayed a period of disrupted behavior. In later experiments, the guillotine door was not lowered and response time continued to be accumulated if the rat reinserted its nose within 140 msec. Due to the uncertainty which the latter procedure introduced concerning the onset of the odorsampling period, such trials were excluded from the quantitative analyses.

Quantitative analyses and statistical treatments. The tape-recorded data were digitized at $128 \mathrm{~Hz}$ for our computer analyses. This report focuses on two 1-sec data epochs from each trial of reversal training. The first epoch, designated approach, began $2 \mathrm{sec}$ before the response onset (breaking of the photocell beam inside of the port) and generally corresponded to the period of whole body locomotion toward the port. The second epoch, designated sampling, began when the photocell beam inside of the port was broken. FFT analyses applied to these 1-sec epochs yielded spectra having a Nyquist frequency of $64 \mathrm{~Hz}$ with $1 \mathrm{~Hz}$ resolution. Amplitude and phase spectra for sniffing and hippocampal RSA were generated with the procedures described by Dumermuth et al. (1972). These spectra were used to calculate the relative amplitude spectra and resultant phase differences across trials as described under "Results." The statistical significance of resultants was evaluated with procedures based on the Rayleigh test (Curray, 1956; Durand and Greenwood, 1958; Zar, 1974).

\section{Results}

This report deals exclusively with data from the first reversal in order to avoid interanimal variations which might be related to differences in the rate of familiarization with the behavioral paradigm during initial discrimination learning or in the rate of learning set formation during subsequent reversals. The typical pattern of performance during the first reversal was as follows. 'The rat initially would continue to perform the previously learned responses and, due to the reversal of stimulus valences, would fail to obtain water rewards. The rat soon reverted to the response pattern acquired during shaping and exhibited $\mathrm{R}+$ responses on nearly all trials.
The latter strategy successfully achieved the average $50 \%$ payoff and was continued for a considerable number of trials. The rat then learned to withdraw its nose from the port in less than $2 \mathrm{sec}$ during presentations of the current $\mathrm{S}-$ stimulus (which now was never followed by rewards) and continued to perform well above chance levels during the period of overtraining.

Qualitative analyses. Figure 2 illustrates sets of records taken from the block of trials in which a rat again began to increase its incidence of $R$ - responses but had not yet achieved criterion level performance. The records are of $4 \mathrm{sec}$ duration and are centered at the beginning of the sampling epoch (upward deflection of the event marker). During the first 1-sec epoch of each set (approach), the hippocampal record contained characteristically high amplitude, relatively fast RSA, and the thermocouple record showed relatively slow breathing. Most of the hippocampal records showed an abrupt decrease in the amplitude and frequency of the RSA shortly before the beginning of the sampling epoch. This change commonly occurred when the animals had reached the port and stood waiting for the guillotine door to open and characteristically continued into the sampling epoch. During the sampling epoch, the rats exhibited high frequency (greater than $4 \mathrm{~Hz}$ ) sniffing. These sniffing bouts typically began upon opening of the guillotine door and before insertion of the nose into the port. High frequency sniffing at the water cup also was common following

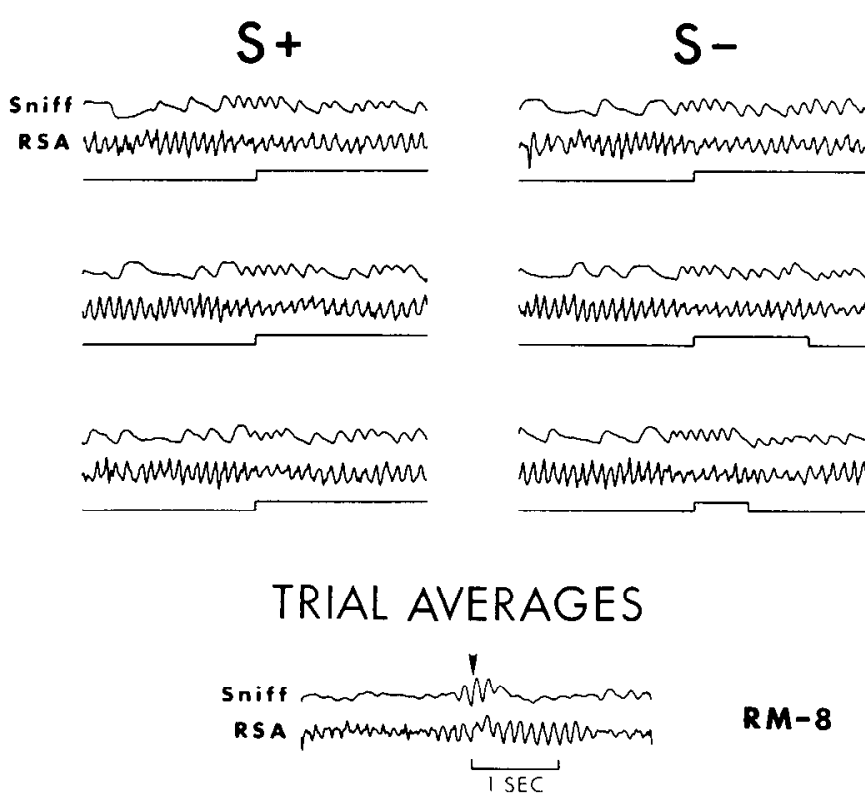

Figure 2. Sample records (top) and averaged sniffing and hippocampal RSA for a block of 40 precriterion trials (bottom) during odor discrimination reversal learning. The raised portions of the event marker records indicate periods when the animal (RM-8) had its snout inserted in the sniff port. The arrowhead identifies the onset of the sniff cycle used to align each record for the trial averages (i.e., the first complete sniff cycle following insertion of the snout into the sniff port). The animal was rewarded if it held its snout inside of the port for at least 2 sec during $S+$ trials and never was rewarded during $S-$ trials. The individual records and trial averages illustrate the tendency of the animal to entrain its sniffing bouts to ongoing trains of hippocampal RSA. 

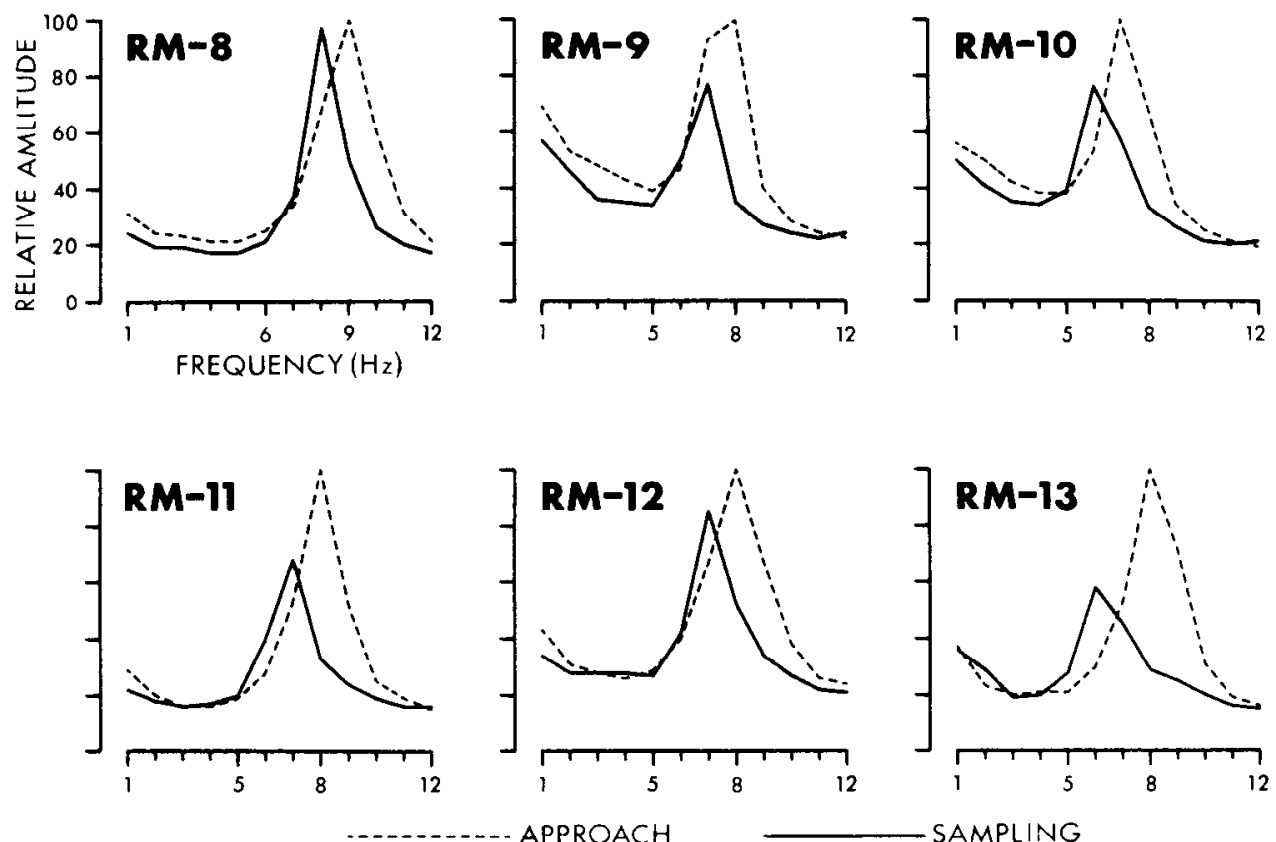

Figure 3. Relative amplitude spectra of hippocampal RSA during the approach and sampling epochs. The spectra for RM-11 are based on 454 trials; the numbers of trials for the other 5 animals are listed in Figure 5 . All 6 of the animals exhibited a tendency toward lower frequency RSA during the sampling epochs.

withdrawal of the nose from the port. Unless the guillotine door already had opened by the time an animal reached the port following initiation of a trial, the sniffing bouts typically did not commence until shortly after the decrease in the amplitude and frequency of hippocampal RSA.

Our qualitative analyses indicated that, during the periods when a rat had its nose inserted in the port and during sniffing at the water cup, the sniffing bouts tended to become entrained to the hippocampal RSA such that individual sniff cycles within a bout occurred with the same repetition rate as individual cycles of the RSA. The repetition rate varied somewhat from one entrainment to another. Also, entrainments were not observed on all trials. They were common during the early, typically unrewarded trials of reversal training before the animals reverted to a predominantly $\mathrm{R}+$ pattern of responding. Entrainments also were common during the early trials of each day and were observed most consistently during the trials shortly preceding the attainment of criterion level performance. The traces at the bottom in Figure 2 illustrate our qualitative analyses using averaging procedures for a block of precriterion trials. 'Ihe first sniff cycle within each sampling epoch was located using a peak detection routine, and the individual trial records were shifted so that these first sniff cycles would be aligned. The thermocouple and the hippocampal record of each set then were summed across trials and divided by a scale factor. The thermocouple average in Figure 2 shows a burst of sniffing distributed about the sniff cycle used to align each record (arrowhead). The hippocampal average shows a train of oscillations approximately corresponding to the period of lower amplitude, slower RSA in the raw data records. This train of averaged RSA extends throughout the period corresponding to the sam- pling epochs and begins before the burst of averaged sniffing. The amplitude of averaged RSA during the yet earlier period corresponding to the approach epochs is reduced markedly, despite the presence of characteristically high amplitude RSA during individual approach epochs in the raw data records. Such averages support our visual impressions from graphic displays of the raw data that $(i)$ the changes in RSA shortly preceded the investigatory sniffing and (ii) during the sampling epochs, individual sniffs tended to occur with a preferred temporal relationship to cycles of ongoing RSA. However, because such averages do not specifically take into account the variations in sniffing and RSA frequencies across trials, they cannot distinguish whether sniffing exhibits a preferred phase or a preferred latency relationship to the RSA.

Quantitative analyses. The apparent differences in the amplitude and frequency of hippocampal RSA during the approach and sampling epochs were quantified with the use of power spectra (cf., Dumermuth et al., 1972). The amplitude components of the individual spectra (i.e., square root of power at each frequency) for each of these two epochs were summed across trials for each animal without normalization, and then a scale factor was applied such that the largest amplitude component in either of the two summed spectra over the $1-$ to $12-\mathrm{Hz}$ band was represented as $100 \%$. These relative amplitude spectra for the approach (dashed lines) and sampling (solid lines) epochs are illustrated in Figure 3. All 6 of the animals exhibited a shift toward lower amplitude, slower RSA during the sampling epoch. For most of these animals, the RSA during the sampling epoch had power predominantly in the 5 - to $8-\mathrm{Hz}$ band with a peak at 6 or $7 \mathrm{~Hz}$. One animal (RM-8) had power predominantly in the $6-$ to $9-\mathrm{Hz}$ band with a peak at $8 \mathrm{~Hz}$. In subsequent 
analyses, the temporal relationship between sniffing and RSA therefore was examined over the 5- to $8-\mathrm{Hz}$ band for 5 of the animals and over the $6-$ to $9-\mathrm{Hz}$ band for RM8.

The subsequent analyses were designed to characterize the parameters of the temporal relationship for individual animals and to generate a measure of its statistical significance across blocks of trials. As can be seen in Figure 2, the waveform of the hippocampal RSA is not strictly sinusoidal but is more similar to a sawtooth and can vary in the sharpness of its peaks and troughs or in the slopes of its rising and falling phases from one wave to the next. The waves in the thermocouple record during investigatory sniffing bouts also exhibit such variations, and the bouts occasionally are mixed with slower breathing (i.e., waves whose periods are outside of the RSA band during the sampling epoch as defined above). Therefore, rather than attempting to analyze the relationship between investigatory sniffing and the hippocampal RSA on a wave-by-wave basis within each train, we employed modeling procedures based on the FFT to minimize errors due to variations in individual waveforms and to obviate the need for judgements as to whether individual cycles in the thermocouple record were or were not part of a sniffing bout. These procedures focused on the cyclic (i.e., repetitive) properties of investigatory sniffing and hippocampal RSA and sought to characterize the temporal relationship between rhythmic sniffing behavior and the pacemaker activity which drives hippocampal RSA during periods of entrainment between the sniffing and limbic $\theta$ rhythms.

To perform the analyses, the phase angle of each frequency component in the power spectra was expressed as the phase of an equivalent sine wave (cf., Dumermuth et al., 1972), and, for each trial, the phase difference between sniffing and RSA during the sampling epoch was modeled as follows. The dominant (highest amplitude) frequency component of sniffing within the chosen frequency band was identified in the power spectrum; the phase difference $\left(0\right.$ to $\left.360^{\circ}\right)$ between sniffing and RSA at this dominant frequency then was determined by subtracting the phase angle of the sniffing component from the phase angle of the RSA component using polar arithmetic. This procedure generated a matrix of phase differences as a function of dominant sniffing frequency across trials for each animal. If a rat did not entrain its sniffing to the limbic $\theta$ rhythm reliably or if a rat's sniffing bouts did tend to adopt the same frequency as the ongoing $\theta$ rhythm but without a preferred phase or latency relationship, then the distributions of phase differences at each frequency within such a matrix would approach randomness. If a rat tended to entrain its sniffing to the $\theta$ rhythm with a preferred phase relationship, the distributions of phase differences within the matrix each would have a peak and would be similar across frequencies. If, as suggested by our previous findings in the hamster (Macrides, 1975; Youngs et al., 1978), the entrainments were characterized by a preferred latency relationship between the onsets of sniff cycles and a particular phase of the pacemaker activity which derives the $\theta$ rhythm and the recorded RSA, the matrix would exhibit a frequency-dependent variation in phase differences, and regression analyses could be performed to determine the latency for each animal. Once the parameters of the temporal relationship were characterized from the overall matrix, an appropriate correlational statistic could be developed to assess the consistency with which an animal exhibited the relationship across any selected blocks of trials.

Analyses of the matrices indicated that the phase differences between sniffing and RSA varied linearly as a function of frequency and thus reflected a preferred latency relationship. This variation is illustrated for animal RM-9 in Figure 4. The distributions of phase differences are plotted as incidence histograms for each frequency. $\chi^{2}$ tests applied to these distributions confirmed that, at each frequency, the rat exhibited a preferred phase relationship between sniffing and RSA $(p<0.001$, $0.001,0.05,0.001$ for 5 to $8 \mathrm{~Hz}$, respectively). It is also apparent from inspection of these histograms that the preferred phase relationship shifted toward progressively smaller phase differences with increasing frequency. This systematic variation was confirmed by computing a resultant phase difference at each frequency and then calculating a least squares line for the four resultants. To compute the resultants, the phase difference for each epoch was treated as a vector with unity amplitude and the vectors were summed using polar arithmetic. For this animal, the preferred phase differences tended to be reduced by $44^{\circ}$ for each $1-\mathrm{Hz}$ increase in frequency as
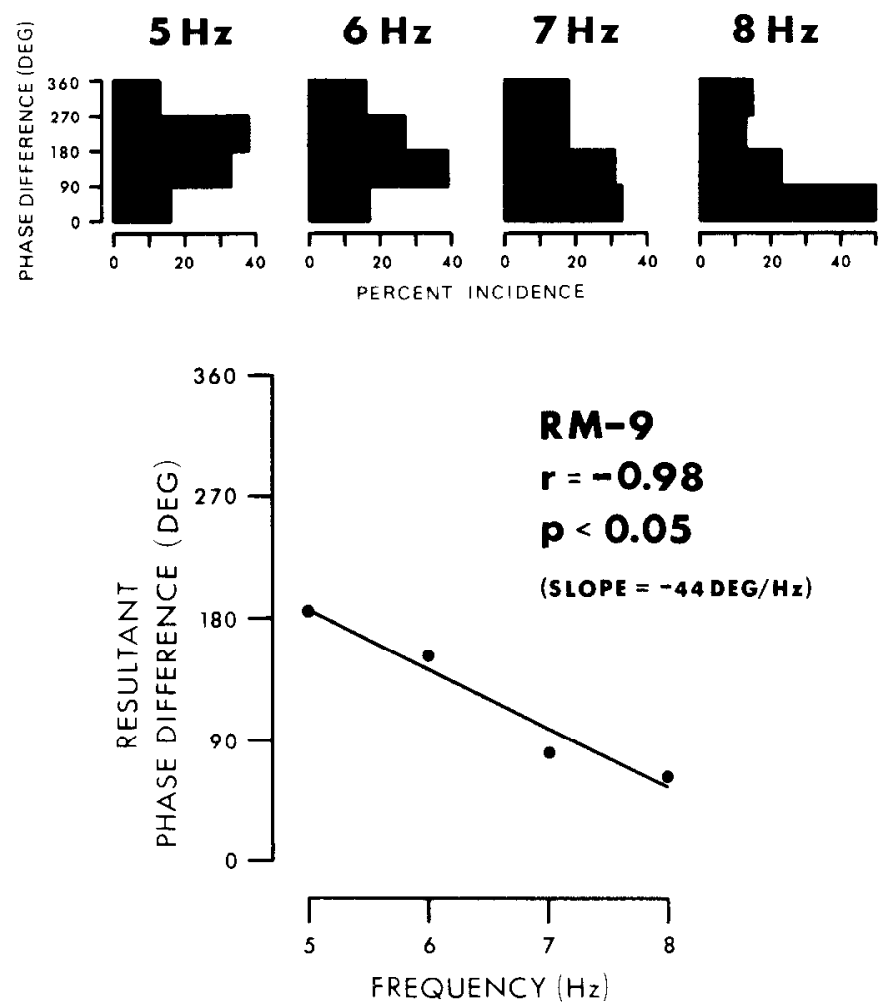

Figure 4. Phase relationship between sniffing and hippocampal RSA as a function of the dominant sniffing frequency during the sampling epochs. At the top, the data from 658 trials for animal RM-9 are plotted as incidence histograms. At the bottom, resultant phase differences (see the text) derived from these same data are plotted as a function of frequency together with the least squares regression line. The preferred phase difference decreased by $44^{\circ}$ for each $1-\mathrm{Hz}$ increase in dominant sniffing frequency. 


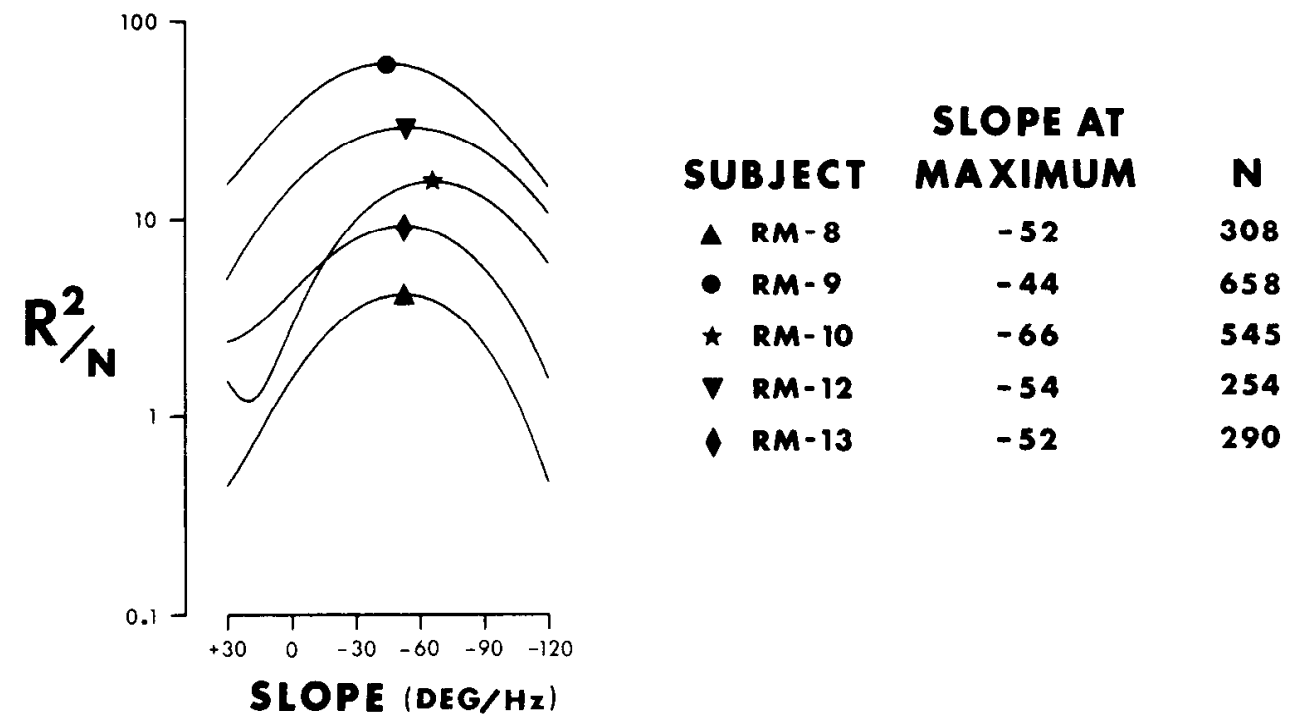

Figure 5. Analysis of the preferred temporal relationship between sniffing and hippocampal RSA based on a modification of the Rayleigh test. The curves on the left were derived by computing the resultant amplitude $(R)$ for each animal at each of 360 assumed slopes of the nature shown in Figure 4 (see text) and are plotted over a range which includes their maxima $(N=$ number of trials for each animal). 'These maxima define the slope which best characterizes the rate of change in preferred phase difference between sniffing and RSA as a function of the dominant sniffing frequency during the sampling epochs.

indicated by the slope of the least squares line (Pearson $r=-0.98 ; p<0.05)$.

The analyses in Figure 4 are presented primarily to illustrate the frequency dependence in the phase difference data and the general principles which underlie the quantitative analyses of the temporal relationship between the sniffing and limbic $\theta$ rhythms. The use of a least squares line to determine the changes in phase difference with frequency is not a powerful procedure because it requires that a data matrix be collapsed into four points and thus does not take into account the number of phase difference measurements at each frequency. Least squares lines cannot be calculated directly from the data matrices because the phase differences have a circular distribution (cf., Youngs et al., 1978, for a nonparametric solution). To illustrate further the principles which underlie our present analyses, consider the following. If we had collapsed the data in Figure 4 across frequencies and plotted a single incidence histogram, the existence of a preferred temporal relationship between sniffing and RSA might have been obscured because the peaks of the phase difference distributions differ across frequencies. However, if we first rotated each data point in the matrix for this animal through $-44^{\circ} / \mathrm{Hz}$, the peaks of the phase difference distributions at each frequency would become aligned. We then could appropriately collapse across frequencies to construct a single incidence histogram or compute an overall resultant for the data matrix. The amplitude of the latter resultant would reflect the strength of the temporal relationship across frequencies and would provide a powerful statistic for assessing whether the distribution of phase differences throughout the matrix differs from that which would be expected by chance (see below). Furthermore, if we rotated each data point through somewhat more or somewhat less than $-44^{\circ} / \mathrm{Hz}$, the peaks of the distributions at each frequency would be less well aligned and the amplitude of the overall resultant would be reduced. Thus, a plot of resultant amplitude as a function of assumed slope (i.e., change in preferred phase difference per $\mathrm{Hz}$ ) should take the form of an inverted $U$ with a maximum at the slope which best characterizes the rate of change in preferred phase difference with dominant frequency. The construction of such a plot and determination of the slope which yields a maximum resultant amplitude is essentially a form of regression analysis.

The latter type of plot was constructed for each data matrix as follows. A series of slopes in the range of $-180^{\circ} / \mathrm{Hz}$ to $179^{\circ} / \mathrm{Hz}$ at $1^{\circ}$ intervals each was assumed, the appropriate rotations of the phase difference measurements for each assumed slope were performed, and the corresponding 360 overall resultant amplitudes $(R)$ each were computed. Because the phase difference measurements have a circular distribution, a slope of $-180^{\circ}$ \% $\mathrm{Hz}$ is equivalent to a slope of $180^{\circ} / \mathrm{Hz}$. One animal (RM11) did not have a significant $R(p<0.05)$ with any degree of rotation (see below). The curves for the other 5 animals are presented in Figure 5 over a range of assumed slopes which includes their maxima. The probability of obtaining a resultant amplitude greater than $R$ by pure chance varies with $e^{-R^{2} / N}$, where $N$ is the number of measurements (see discussion of the Rayleigh test in Curray, 1956). The data therefore are plotted as $R^{2} / N$ on a logarithmic scale so that the ordinates reflect the relative significance levels of the resultant amplitudes. 'The maximum for each animal is indicated on the appropriate curve, and the corresponding slope is listed at right in Figure 5. Across the 5 animals, these slopes of best fit ranged from $-44^{\circ} / \mathrm{Hz}$ to $-66^{\circ} / \mathrm{Hz}$ (mean $=-54^{\circ}$ / $\mathrm{Hz})$ and their significance levels ranged from $p<0.02$ (RM-8) to $p<10^{-26}$ (RM-9). The slopes of best fit corresponded to those obtained with the procedures illustrated in Figure 4, but, as expected, the procedures in Figure 5 were more powerful in that they yielded more extreme significance levels.

The phase angle of the resultant with maximum am- 
plitude is formally equivalent to the $y$ intercept in a linear regression analysis (i.e., in a relationship which could be described by an equation with the general form, $y=b+m \cdot x)$. We will refer to this phase angle as the constant, $\Phi_{r}$. We will refer to the preferred phase difference al any frequency in the sniffing and RSA band as the variable, $\Phi_{0}(f)$, where $f$ is the frequency. Because of the direction of subtraction used in our phase difference measurements, $\Phi_{0}(f)$ is equivalent to the phase of RSA at the defined onsets (zero phases) of sniff cycles which exhibit the preferred temporal relationship between sniffing and RSA. Our analyses support the interpretation that $\Phi_{0}(f)$ decreases linearly across the examined frequencies in the sniffing and RSA band. The preferred phase difference at any of these frequencies therefore can be described by the equation

$$
\Phi_{11}(f)=\Phi_{\tau}+\text { slope } \cdot f+n \cdot 360
$$

where slope is the slope of best fit and $n$ is 0 or an integer such that $\Phi_{0}(f)$ falls in the range of 0 to $360^{\circ}$. The experimentally determined negative quantity, slope, can be used to calculate a positive quantity, $\tau$, from the equation

$$
\text { slope } \mathrm{deg} / \mathrm{Hz}=-\tau \text { msec } \cdot 360^{\circ} / \text { cycle } \div 1000 \mathrm{msec} / \mathrm{sec}
$$

and the preceding equation can be expressed as

$$
\Phi_{0}(f)=\Phi_{\tau}-0.36 \cdot \tau \cdot f+n \cdot 360
$$

The latter equation directly describes the temporal relationship between sniffing and RSA during entrainments as exhibiting a preferred latency, where $\tau$ is this latency in milliseconds. The preferred latency relationship is modeled in Figure 6 using experimentally determined parameters from 3 animals. The slopes of best fit and the corresponding values of $\tau$ for these 3 rats span the range of values for animals with significant maximum resultant amplitudes (mean value of $\tau=149 \mathrm{msec} ; N=$ 5). As is illustrated in Figure 6, the phase difference between sniffing and RSA varies across frequencies in a manner such that, regardless of the frequency, the zero phase of each sniff cycle occurs $\tau$ msec before the particular phase, $\Phi_{\tau}^{\circ}$, of the RSA.

As is also illustrated in Figure 6, the experimentally determined values of $\Phi_{\tau}$ differed considerably for different animals. The RSA recorded in the hippocampal formation exhibits phase reversals when an electrode tip crosses the stratum pyramidale in the hippocampus or the stratum granulosum in the dentate gyrus (cf., Winson, 1974, 1976). If the preferred latency relationship were such that the animals limed their sniffs relative to a uniform phase of the pacemaker activity which drives the limbic $\theta$ rhythm and the recorded RSA, then the experimentally determined values of $\Phi_{\tau}$ would vary across animals in relation to the locations of the electrode tips in the hippocampal formation. Figure 7 shows the locations of the noninverting tips (solid circles) and inverting tips (open circles) for the 6 animals. For the rat which did not exhibit a significant relationship between sniffing and RSA (RM-11), the noninverting tip had passed through the dentate gyrus and neither tip of the electrode was positioned in the hippocampal formation. At the upper right in Figure 7 , the locations of the
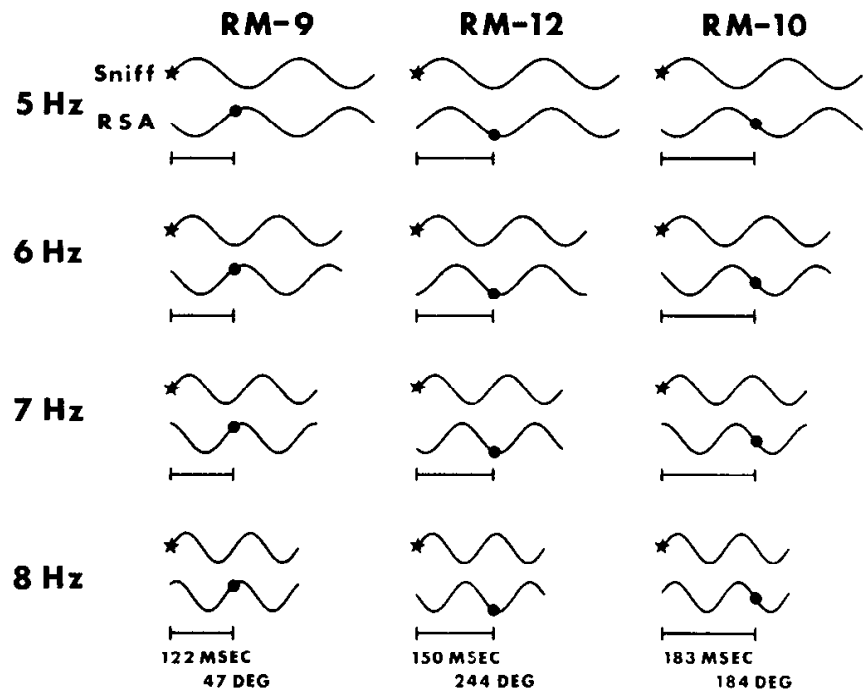

Figure 6. Model of the preferred temporal relationship between sniffing and the limbic $\theta$ rhythm. Across frequencies, the phase difference between sniffing and hippocampal RSA varies in a manner such that the zero phases of sniff cycles (stars) occur at a preferred interval, $\tau$ msec (bars), before the particular phase, $\Phi_{\tau}^{\circ}$ (solid circles), of the RSA. This latency relationship is illustrated for 3 animals using sinusoidal waves and experimentally determined values of $\tau$ and $\Phi_{\tau}$ (bottom).

noninverting tips for the other 5 animals are shown schematically relative to the strata pyramidale and granulosum. A series of five sinusoidal waves having equal periods are drawn at the lower right. Fach wave has a symbol demarking $\Phi_{\tau}$ for 1 of the animals, and the waves have been drawn with these phases aligned vertically. When the waves are so aligned, they show the kinds of phase reversal that would be expected from the variations in recording tip location. Our results therefore are consistent with the interpretation that, during the sampling epochs, these animals exhibited entrainments with a preferred latency relationship between the onsets of their sniff cycles and a particular phase of the pacemaker activity which drives the limbic $\theta$ rhythm.

Our impression from graphic displays of the raw data was that the consistency with which the animals exhibited this relationship varied during the course of training (see above). To evaluate the variation, the trials were grouped into blocks of 40 and the resultant amplitudes were calculated for the sampling epochs using each rat's slope of best fit. Figure 8 shows the variations in resultant amplitude (expressed as $R^{2} / 40$ ) for the two blocks which immediately preceded the achievement of criterion level performance (solid bars) and for the block which includes the criterion run and the immediately following 20 trials of overtraining (open bars). Across animals, the resultant amplitudes were consistently high for the block of 40 trials which preceded the criterion run, and all of the animals showed some increase from the yet earlier block of 40 trials. Four of the 5 animals showed a decrease in resultant amplitude during the criterion and overtraining block, and in 3 of the animals this decrease was appreciable. Also, the 2 animals which did not exhibit an appreciable decrease (RM-9 and RM-10) were the slowest to acquire the reversal (cf., number of trials in Fig. 5), 
and both animals exhibited a weakening of the relationship during later trials of overtraining. The tendency of the animals to exhibit the preferred latency relationship thus was not associated specifically with correct performance. Rather, the preferred latency relationship was observed more reliably during periods when the animals were most likely to be evaluating the behavioral relevance of the stimuli and were in the process of modifying their behavior in relation to those stimuli.

\section{Discussion}

Previous analyses of the $\theta$ rhythm have focused either on the gross behavioral correlates of its presence or absence (cf., Vanderwolf, 1975; Berry and Thompson, 1978 ) or on the liming of specific neural or behavioral events as a function of $\theta$ cycle phase (cf., Komisaruk, 1977). We have attempted to join these two concerns by creating a behavioral paradigm which allows repetitive observation of both gross and cyclic temporal relationships between selected behaviors and the $\theta$ rhythm during the acquisition of learned responses and performance of a sensory discrimination (cf., Eichenbaum, 1981). Relatively high frequency, large amplitude RSA is associated with gross locomotory approach behavior in our task. Since Vanderwolf's (1969) initial report, numerous investigators have confirmed the association of hippocampal $\theta$ activity with locomotion in a variety of experimental situations (cf., Vanderwolf and Robinson, 1981). During odor sampling in our task, the rats are immobile except for those movements associated with sniffing behavior. Hippocampal RSA is present reliably during these pe- riods and tends to be lower in frequency and amplitude than the RSA recorded during locomotory approach. Hippocampal RSA has not commonly been observed during relative immobility in waking rats but has been demonstrated previously to occur during relative immobility in other learning paradigms specifically designed to discourage or delay the locomotory behavior or gross bodily movements of the rats (Vanderwolf, 1969, 1975; Whishaw, 1972). Hippocampal RSA is observed more

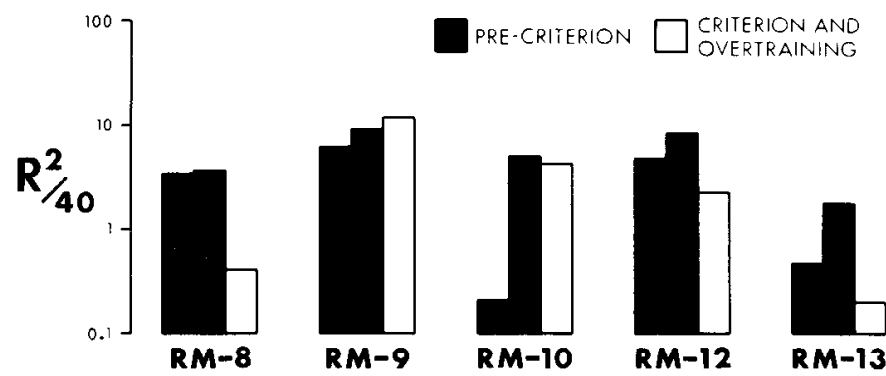

Figure 8. Variations in the consistency of the preferred latency relationship between sniffing and the $\theta$ rhythm during the sampling epochs across successive blocks of 40 trials. Resultant amplitudes $(R)$ were calculated using each animal's slope of best fit. In 4 of the 5 animals, the highest degree of consistency was exhibited during the 40 trials immediately preceding the achievement of criterion level performance. Three of the 5 animals exhibited appreciably lower consistency of the relationship during the criterion and overtraining block, indicating that the relationship was more prevalent when animals were in the process of modifying their responses to discriminant odor stimuli.
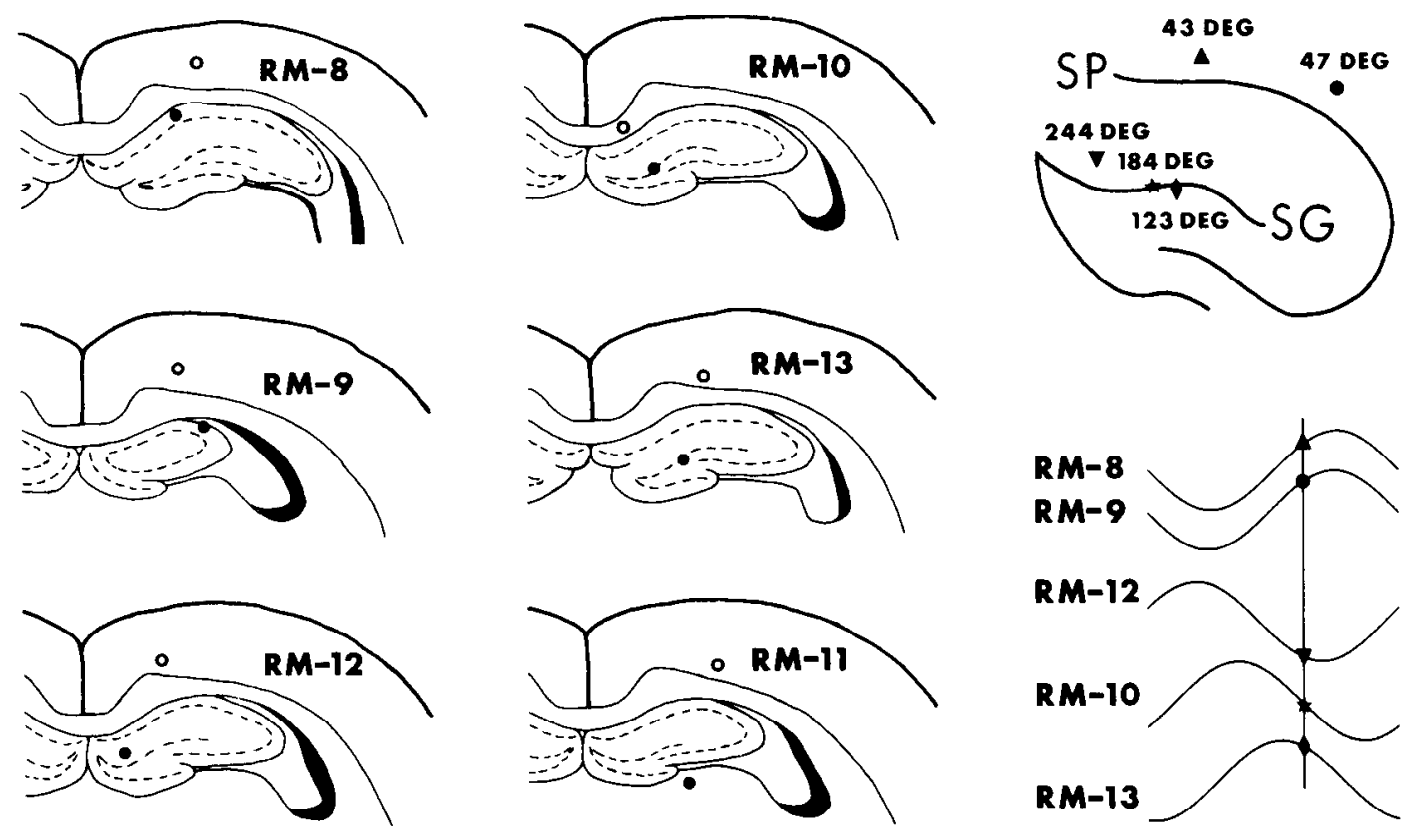

Figure 7. Relationship between hippocampal electrode placements and values of $\Phi_{r}$. The locations of the noninverting (solid circles) and inverting (open circles) electrode tips are indicated for each of the 6 animals. At the upper right, the locations of the noninverting tips relative to the stratum pyramidale $(S P)$ and stratum granulosum $(S G)$ are shown together with the experimentally determined values of $\Phi_{\tau}$ for the 5 animals which exhibited significant correlations between sniffing and hippocampal RSA. At the lower right, a series of five sinusoidal waves aligned with respect to $\Phi_{\tau}$ illustrate depth-related phase reversals as would be expected if these animals were exhibiting a preferred latency relationship to a uniform phase of the pacemaker activity which drives the $\theta$ rhythm and the recorded RSA. 
readily during relative immobility in waking rabbits, cats, and dogs and during urethane- or ether-induced anesthesia in the rat (cf., Robinson, 1980). The hippocampal RSA which normally occurs during relative immobility in the waking state or during ether- or urethane-induced anesthesia has been shown to be blocked by atropine in both the rabbit and the rat, and, in both species, an atropine-insensitive RSA of generally higher frequency is present during motor patterns such as walking, head movements, or rearing (Kramis et al., 1975; Vanderwolf, 1975). The differences in the spectral characteristics of the RSA recorded during the approach and sampling epochs in our task may reflect differences in the dependence of the RSA on the integrity of central cholinergic mechanisms but also could be related to other variables, such as the vigor of motor activity (Whishaw and Vanderwolf, 1973).

Our analyses further indicate that, during the sampling epochs, rhythmic sniffing tends to occur with a preferred latency relationship to the limbic $\theta$ rhythm. This tendency is particularly strong shortly before the attainment of criterion level performance. The timing of investigatory sniffs as a function of $\theta$ cycle phase thus may be important for neural processing of sensory and/or motor information of relevance for response modification. The olfactory bulb and olfactory cortices have heavy projections to the lateral entorhinal cortex (Davis et al., 1978;
Haberly and Price, 1978a, b; Kosel et al., 1981), which in turn projects to the dentate gyrus, hippocampus, and subiculum (Steward, 1976; Steward and Scoville, 1976). The temporal relationship may provide a neural basis for modulating olfactory input to the hippocampal formation when an animal is evaluating the behavioral relevance of odors (cf., Macrides, 1976; Komisaruk, 1977; Rudell et al., 1980; Deadwyler et al., 1981). It should be stressed, however, that the preferred latency relationship need not be important for information processing solely within the hippocampal formation. Our analyses of this relationship as a function of recording tip location within the hippocampal formation indicate that the timing of sniffs is correlated with the pacemaker activity in the medial septum-diagonal band complex which drives the hippocampal RSA (cf., Petsche et al., 1962, 1965). As illustrated in Figure 9, recent anatomical studies have demonstrated projections from the medial septum-diagonal band complex to the main olfactory bulb (MOB) and to the cortical olfactory, amygdaloid, and parahippocampal regions which receive inputs from the MOB as well as extensive interconnections among these cortical structures and the hippocampal formation (Beckstead, 1978; Davis et al., 1978; Davis and Macrides, 1981; Haberly and Price, 1978a, b; Luskin and Price, 1982; Macrides et al., 1981; Macrides and Davis, 1983; Mitchell et al., 1982; Sørensen and Shipley, 1979; Swanson and Cowan, 1977). In record-

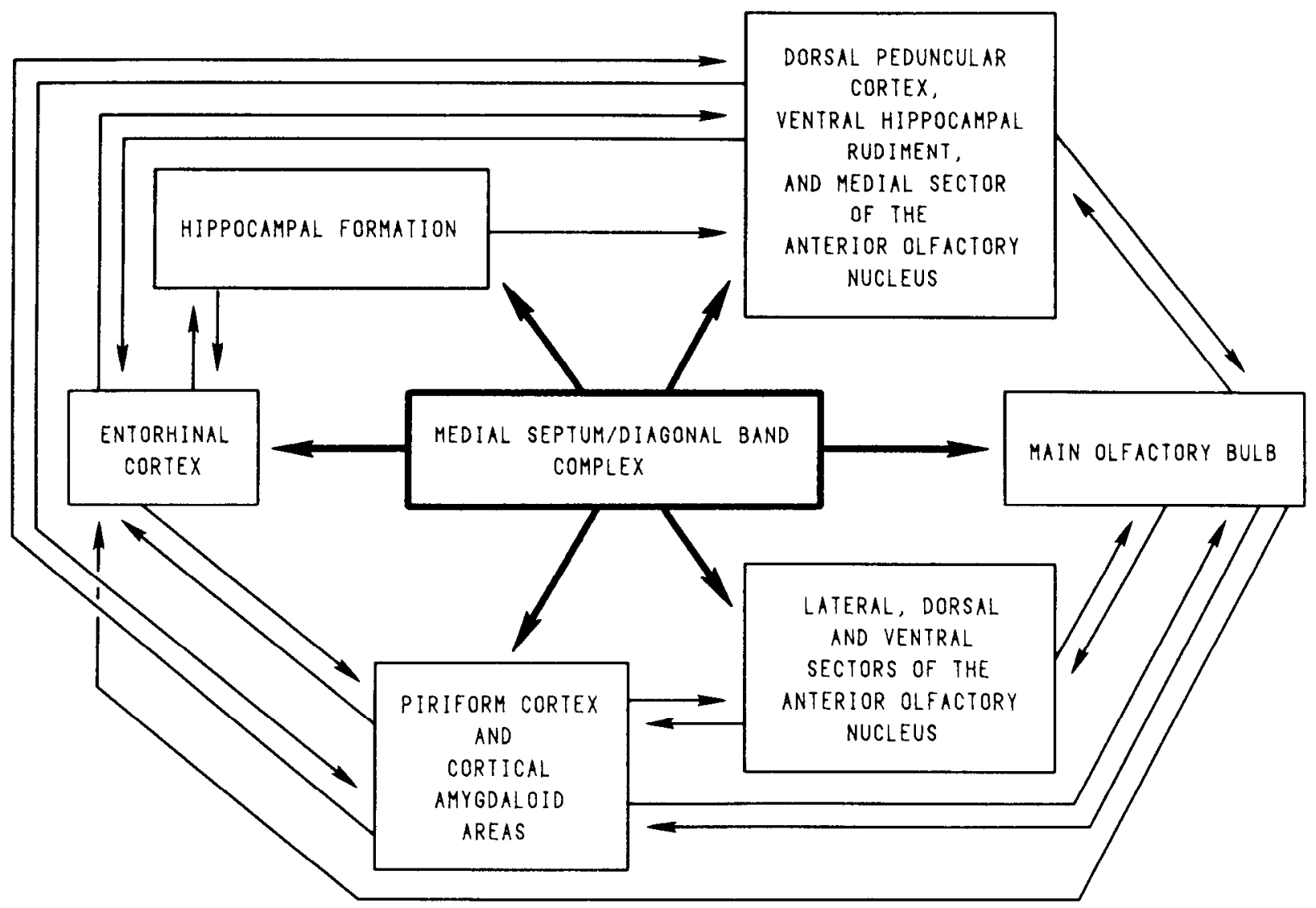

Figure 9. Schematic diagram of the interconnections among olfactory and limbic cortical structures and projections to these structures from the medial septum-diagonal band complex. By virtue of its projections, the medial septum-diagonal band complex may provide $\theta$-related modulation of information processing throughout the olfactory system and the related structures of the limbic system. 
ings from paralyzed animals which have their normally pulsatile nasal airflow interrupted, we have observed strong correlations between the bursting activity of single MOB units and individual cycles of the hippocampal RSA (Macrides et al., 1979). These anatomical and electrophysiological findings suggest that the pacemaker system for hippocampal RSA also drives $\theta$ activity in the olfactory system, and thus the timing of sniffs relative to $\theta$ cycle phase may be important for the modulation of information processing throughout the olfactory system and the related structures of the limbic system.

Our finding that the onsets of sniff cycles tended to lead a phase of the recorded RSA $\left(\Phi_{\tau}\right)$ raises concern that the hippocampal recordings may have represented sensory and/or motor evoked responses either occurring within the hippocampal formation or volume-conducted from other brain structures and thus might not have been a valid monitor of the limbic $\theta$ rhythm. Our failure to find a significant correlation for the animal whose noninverting recording tip had passed through the hippocampal formation and our finding that, for the animals with significant correlations, the values of $\Phi_{\tau}$ varied as would be expected based on the locations of the recording tips relative to the phase reversal regions within the hippocampal formation provide compelling evidence that, in the latter animals, our recordings predominantly represented endogenous hippocampal activity. Also, our observation that trains of averaged RSA tended to begin before and to be of longer duration than trains of averaged sniffing during the periods of odor sampling (cf., Fig. 2) increases our confidence that the quantitative analyses characterized the relationship between bouts of investigatory sniffing and ongoing trains of hippocampal RSA (i.e., if the sniffing were eliciting the RSA or resetting its phase, the trains of averaged RSA would not have commenced before the trains of averaged sniffing). Based on the anatomical interconnections illustrated in Figure 9, it would be surprising if odor-evoked and/or motor-related neuronal activity in the hippocampal formation were not at all affected by the temporal patterns of investigatory sniffing. Indeed, the trains of averaged RSA typically were superimposed on a baseline shift following the onset of, and lasting through, the trains of averaged sniffing (cf., Fig. 2). This slow potential probably represents an evoked response associated with the sniffing bouts. However, because we saw no consistent changes in the amplitude of averaged RSA shortly following the trains of averaged sniffing and this slow potential and because, in the raw data records, we saw no obvious relationship between the amplitude of the RSA and the presence or absence of entrained sniffing, the evoked sensory and/or motor responses do not appear to have been reflected prominently within the RSA frequency band. $\theta$-related variations in the amplitude of hippocampal evoked population potentials following electrical stimulation of hippocampal afferents as reported by Rudell et al. (1980) may be due to an extraordinary synchrony in the afferent volley and may be influcnced by concomitant antidromic stimulation of efferent projections from the hippocampal formation or from the medial septum-diagonal band complex (cf., Fig. 9 ). Under more natural conditions, $\theta$-related modulation of afferent input to the hippocampal formation might alter the spatial and/or temporal patterns, but not the overall synchrony, of evoked neuronal activity. The finding of Buño et al. (1978) that electrical stimulation of the entorhinal cortex can reset the phase of hippocampal RSA also may be related to concomitant antidromic stimulation of hippocampal efferents and of the $\theta$ pacemaker system in the medial septum-diagonal band compex. The hippocampal recordings thus can be assumed to have provided a valid, albeit indirect, monitor of the endogenous pacemaker activity which drives the limbic $\theta$ rhythm.

The present findings are consistent with Komisaruk's (1970) speculation that temporal regulation of exploratory movements is important for the processing of sensory inputs to the limbic-hypothalamic system and with our speculation (Macrides and Chorover, 1972) that the cyclic aspects of olfactory sampling play a role in olfactory processing. The definition of sniff cycle onset in our analyses is arbitrary. When animals exhibit entrainments with the preferred latency relationship, individual cycles of the sniffing rhythm can be conceptualized as leading individual cycles of the $\theta$ rhythm by approximately 150 $(\tau)$ msec, and thus, any arbitrarily selected phase in the sniff cycle exhibits this preferred latency relationship to a respective phase of the $\theta$ rhythm. Furthermore, the experimentally determined values of $\tau$ would be expected to vary somewhat as a function of the anterior-posterior placement of the thermocouple in the nasal cavity, because the placement affects the time during the sniff cycle when newly inhaled air first reaches the thermocouple and produces measurable cooling (Macrides, 1975). Our single unit studies in rodents indicate that many neurons in the MOB exhibit inhalation-related bursting activity, that the timing of these bursts relative to the onsets of airflow into the nasal cavity can vary by hundreds of milliseconds depending on the odorants in the inhaled air, and that different MOB units can exhibit markedly different burst latencies for any particular odorant or mixture of odorants (Macrides and Chorover, 1972; Macrides, 1976, 1977). Therefore, although, when animals in this study exhibited the preferred latency relationship, the defined onsets of sniff cycles occurred before a particular phase of the $\theta$ rhythm, the excitatory responses of central neurons are likely to have been distributed both before and after this $\theta$ cycle phase. During entrainments, the $\theta$ rhythm might function as a coherent time base for central olfactory and limbic structures and thereby facilitate analysis of odor-induced variations in the timing of neuronal activity relative to the sniff cycles (cf., Macrides, 1975).

\section{References}

Adey, W. R., C. W. Dunlop, and C. E. Hendrix (1960) Hippocampal slow waves: Distribution and phase relations in the course of approach learning. Arch. Neurol. 3: 74-90.

Beckstead, R. M. (1978) Afferent connections of the entorhinal area in the rat as determined by retrograde cell labeling with horseradish peroxidase. Brain Res. 152: 249-264.

Bennett, T. L. (1975) The electrical activity of the hippocampus and processes of attention. In The Hippocampus, R. L. Isaacson and K. Pribram, eds., pp. 71-99, Plenum Press, New York. 
Berry, S. D., and R. F. Thompson (1978) Prediction of learning rate from the hippocampal electroencephalogram. Science 200: 1298-1300.

Buño, W., and J. C. Velluti (1977) Relationships of hippocampal theta cycles with bar pressing during self-stimulation. Physiol. Behav. 19: 615-621.

Buño, W., J. L. Garcia-Sanchez, and E. Garcia-Austt (1978) Reset of hippocampal rhythmic activities by afferent stimulation. Brain Res. Bull. 3: 21-28.

Curray, J. R. (1956) The analysis of two dimensional orientation data. J. Geol. 64: 117-131.

Davis, B. J., and F. Macrides (1981) The organization of centrifugal projections from the anterior olfactory nucleus, ventral hippocampal rudiment, and piriform cortex to the main olfactory bulb in the hamster: An autoradiographic study. J. Comp. Neurol. 203: 475-493.

Davis, B. J., F. Macrides, W. M. Youngs, S. P. Schneider, and D. L. Rosene (1978) Efferents and centrifugal afferents of the main and accessory olfactory bulbs in the hamster. Brain Res. Bull. 3: 59-72.

Deadwyler, S. A., M. West, and J. H. Robinson (1981) Entorhinal and septal inputs differentially control sensory-evoked responses in the rat dentate gyrus. Science 211: 1181-1183.

Dumermuth, G., W. Walz, G. Scollo-Lavizzari, and B. Kleiner (1972) Spectral analysis of EEG activity in different sleep stages. Eur. Neurol. 7: 265-296.

Durand, D., and J. A. Greenwood (1958) Modifications of the Rayleigh test for uniformity in analysis of two dimensional orientation data. J. Geol. 66: 229-238.

Eichenbaum, H. (1981) A behaviorist in the neurophysiology lab. Behav. Brain Sci. 4: 480.

Eichenbaum, H., K. J. Shedlack, and K. W. Eckmann (1980) Thalamocortical mechanisms in odor guided behavior. I. Effect of lesions of the mediodorsal thalamic nucleus and frontal cortex on olfactory discrimination in the rat. Brain Behav. Evol. 17: 255-275.

Gray, J. A. (1971) Medial septal lesions, hippocampal theta rhythm and control of vibrissal movement in the freely moving rat. Electroencephalogr. Clin. Neurophysiol. 30: 169197.

Haberly, L. B., and J. L. Price (1978a) Association and commissural fiber systems of the olfactory cortex of the rat. I. Systems originating in the piriform cortex and adjacent areas. J. Comp. Neurol. 178: 711-740.

Haberly, L. B., and J. L. Price (1978b) Association and commissural fiber systems of the olfactory cortex of the rat. II. Systems originating in the olfactory peduncle. J. Comp. Neurol. 178: 781-808.

Komisaruk, B. R. (1970) Synchrony between limbic system theta activity and rhythmical behavior in rats. J. Comp. Physiol. Psychol. 70: 482-492.

Komisaruk, B. R. (1977) The role of rhythmical brain activity in sensorimotor integration. In Progress in Psychobiology and Physiological Psychology, J. M. Sprague and A. N. Epstein, eds., Vol. 7, pp. 55-90, Academic Press, New York.

Komisaruk, B. R., and C. Beyer (1972) Responses of diencephalic neurons to olfactory bulb stimulation, odors and arousal. Brain Res. 36: 153-170.

Kosel, K. C., G. W. Van Hoesen, and J. R. West (1981) Olfactory bulb projections to the parahippocampal area of the rat. I. Comp. Neurol. 198: 467-482.

Kramis, R. C., C. H. Vanderwolf, and B. H. Bland (1975) Two types of hippocampal rhythmic slow activity in both the rabbit and the rat: Relations to behavior and effects of atropine, diethylether, urethane, and pentobarbitol. Exp. Neurol. 49: 58-85.

Landfield, P. W., J. L. McGaugh, and R. J. Tusa (1972) Theta rhythm: A temporal correlate of memory storage processes in the rat. Science 175: 87-89.
Luskin, M. B., and J. L. Price (1982) The distribution of axon collaterals from the olfactory bulb and the nucleus of the horizontal limb of the diagonal band to the olfactory cortex, demonstrated by double retrograde labeling techniques. J. Comp. Neurol., 209: 249-263.

Macrides, F. (1975) Temporal relationships between hippocampal slow waves and exploratory sniffing in hamsters. Behav. Biol. 14: 295-308.

Macrides, F. (1976) Olfactory influences on neuroendocrine functions in mammals. In Mammalian Olfaction, Reproductive Processes, and Behavior, R. L. Doty, ed., pp. 29-65, Academic Press, New York.

Macrides, F. (1977) Dynamic aspects of central olfactory processing. In Chemical Signals in Vertebrates, D. MullerSchwarze and M. M. Mozell, eds., pp. 499-514, Plenum Press, New York.

Macrides, F., and S. L. Chorover (1972) Olfactory bulb units: Activity correlated with inhalation cycles and odor quality. Science 175: 84-87.

Macrides, F., and B. J. Davis (1983) The olfactory bulb. In Chemical Neuroanatomy, P. Emson, ed., Raven Press, New York, in press.

Macrides, F., A. Bartke, F. Fernandez, and W. D'Angelo (1974) Effect of exposure to vaginal odor and receptive females on plasma testosterone in the male hamster. Neuroendocrinology 15: 355-364.

Macrides, F., P. A. Johnson, and S. P. Schneider (1977) Responses of the male golden hamster to vaginal secretion and dimethyl disulfide: Attraction versus sexual behavior. Behav. Biol. 20: 377-386.

Macrides, F., W. M. Youngs, and B. J. Davis (1979) Relationship between the limbic theta rhythm and ncural activity in the olfactory bulb. Soc. Neurosci. Abstr. 5: 917.

Macrides, F., B. J. Davis, W. M. Youngs, N. S. Nadi, and F. L. Margolis (1981) Cholinergic and catecholaminergic afferents to the olfactory bulb in the hamster: A neuroanatomical, biochemical and histochemical investigation. J. Comp. Neurol. 203: 495-514.

Mitchell, S. J., J. N. P. Rawlins, O. Steward, and D. S. Olton (1982) Medial septal area lesions disrupt $\theta$ rhythm and cholinergic staining in medial entorhinal cortex and produce impaired radial arm maze behavior in rats. .J. Neurosci. 2: 292-302

Murphy, M. R. (1973) Effect of female hamster vaginal discharge on the behavior of male hamsters. Behav. Biol. 9: $367-375$.

O'Keefe, J., and L. Nadel (1978) The Hippocampus as a Cognitive Map, Clarendon Press, Oxford.

Petsche, H., C. Stumpf, and G. Gogolak (1962) The significance of the rabbit's septum as a relay station between the midbrain and the hippocampus. The control of hippocampus arousal by the septum cells. Electroencephalogr. Clin. Neurophysiol. 14: 202-211.

Petsche, H., G. Gogolak, and P. A. van Zwieten (1965) Rhythmicity of septal cell discharges at various levels of reticular excitation. Electroencephalogr. Clin. Neurophysiol. 19: 1624.

Robinson, T. E. (1980) Hippocampal rhythmic slow activity (RSA; theta): A critical analysis of selected studies and discussion of possible species differences. Brain Res. Rev. 2: 69-101.

Rudell, A., S. Fox, and J. R. Ranck, Jr. (1980) Hippocampal excitability phase-locked to theta rhythm in walking rats. Exp. Neurol. 68: 87-96.

Scott, J. W. (1977) A measure of extracellular unit responses to repeated stimulation applied to observations of the time course of olfactory responses. Brain Res. 132: 247-258.

Semba, K., and B. R. Komisaruk (1978) Phase of theta activity in relation to different limb movements in awake rats. Elec- 
troencephalogr. Clin. Neurophysiol. 44: 61-71.

Sørensen, K. E., and M. T. Shipley (1979) Projections from the subiculum to the deep layers of the ipsilateral presubicular and entorhinal cortices in the guinea pig. J. Comp. Neurol. 188: 313-334.

Steward, O. (1976) Topographic organization of the projections from the entorhinal area to the hippocampal formation of the rat. J. Comp. Neurol. 167: 285-314.

Steward, O., and S. A. Scoville (1976) Cells of origin of the entorhinal cortical afferent connections of the hippocampal formation in the rat. J. Comp. Neurol. 172: 49-84.

Swanson, L. W., and W. M. Cowan (1977) An autoradiographic study of the organization of the efferent connections of the hippocampal formation in the rat. J. Comp. Neurol. 172: 4984.

Vanderwolf, C. H. (1969) Hippocampal electrical activity and voluntary movement in the rat. Electroencephalogr. Clin. Neurophysiol. 26: 407-418.

Vanderwolf, C. H. (1971) Limbic-diencephalic mechanisms of voluntary movement. Psych. Res. 78: 83-113.

Vanderwolf, C. H. (1975) Neocortical and hippocampal activation in relation to behaviour: Effects of atropine, eserine, phenothiazines, and amphetamine. J. Comp. Physiol. Psychol. 88: 300-323.

Vanderwolf, C. H., and T. E. Robinson (1981) Reticulo-cortical activity and behavior: A critique of the arousal theory and a new synthesis. Behav. Brain Sci 4: 459-514.
Vertes, R. P. (1981) An analysis of brain stem systems involved in hippocampal synchronization and desynchronization. J. Neurophysiol. 46: 1140-1159.

Welker, W. I. (1964) Analysis of snitting of the albino rat. Behaviour 22: 223-244.

Whishaw, I, Q. (1972) Hippocampal electroencephalographic activity in the Mongolian gerbil during natural behaviours and wheel running and in the rat during wheel running and conditioned immobility. Can. J. Psychol. 26: 219-239.

Whishaw, I. Q., and C. H. Vanderwolf (1973) Hippocampal EEG and behaviour: Changes in amplitude and frequency of RSA (theta rhythm) associated with spontaneous and learned movement patterns in rats and cats. Behav. Biol. 8: 461-484.

Winson, J. (1974) Patterns of hippocampal theta rhythm in the freely-moving rat. Electroencephalogr. Clin. Neurophysiol. 36: 291-301.

Winson, J. (1976) Hippocampal theta rhythm: II. Depth profiles in the freely moving rabbit. Brain Res. 103: 71-79.

Youngs, W. M., W. B. Forbes, and F. Macrides (1978) High coherence between limbic theta rhythm and sniffing in the hamster: Implications for olfactory-limbic integration and hormonal regulation. In Proceedings of the Sixth New Eng. land Bioengineering Conference, D. Jaron, ed., pp. 274-277, Pergammon Press, New York.

Zar, J. H. (1974) Biostatistical Analysis, pp. 310-326, PrenticeHall, Englewood Cliffs, NJ. 